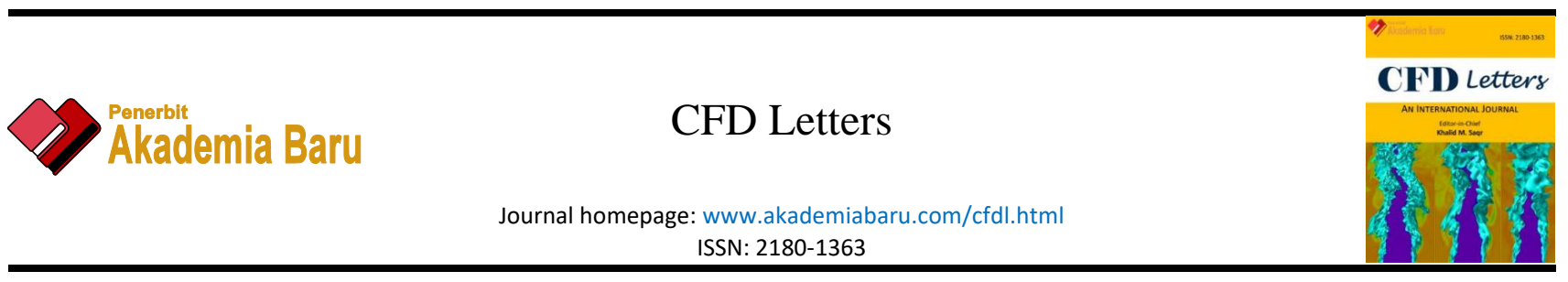

\title{
Numerical Performance Study for the Buoy Shape of Point Absorber Wave Energy Converters
}

\author{
Wan Ching $\operatorname{Tan}^{1}$, Keng Wai Chan ${ }^{1,}{ }^{*}$, Bee Mun Oon ${ }^{1}$, How Tion Puay ${ }^{2}$, Yi Leang Lim ${ }^{1}$ \\ School of Mechanical Engineering, Engineering Campus, Universiti Sains Malaysia, 14300 Nibong Tebal, Penang, Malaysia \\ 2 River Engineering and Urban Drainage Research Centre (REDAC), Engineering Campus, Universiti Sains Malaysia, 14300 Nibong Tebal, Penang, \\ Malaysia
}

\section{\begin{tabular}{l} 
ARTICLE INFO ABSTRACT \\
\hline
\end{tabular}}

Article history:

Received 21 April 2020

Received in revised form 18 June 2020

Accepted 25 June 2020
Available online 30 June 2020

\begin{abstract}
The ocean wave is among the most promising renewable energy available. However, the idea of commercializing wave farm requires high operating cost compared to other RE sources. Effectiveness of wave energy collection device is highly dependent on the location and condition of the wave. Three-dimensional simulation tool is an ideal tool at preliminary design stage to optimise the device. In this paper, a wave structure interaction with application to wave energy device was studied numerically. The computational fluid dynamic analysis based on the Reynolds Average Navier-Stokes equations was used to investigate the interaction between wave and structure, and array effects among devices. Validation and predication of the performance of wave point absorber were done in this paper. Five different models were chosen as the object to study. The influence of wave point absorber devices array on their performance was investigated under the irregular wave conditions to improve the overall performance. Results show that optimum phase condition of buoys can be obtained by adjusting the mass density and radius. Studies found that cylindrical buoy of mass density of $100 \mathrm{~kg} / \mathrm{m}^{3}$ with $0.2 \mathrm{~m}$ radius is the optimal size for the condition set in this research with the produced maximum force of $136.49 \mathrm{~N}$.
\end{abstract}

Keywords:

Buoy; point absorber; CFD; numerical

Copyright $@ 2020$ PENERBIT AKADEMIA BARU - All rights reserved

\section{Introduction}

The ocean wave is among the most promising renewable energy (RE) available. Galarraga stated that the ocean wave is feasible to generate up to 10TW energy with the highest density around [1]. The power density of wave energy is better compared to wind and solar energy. Wave energy converters (WEC) can produce as much as $90 \%$ of the time in a day while solar and wind power systems can produce power around $30 \%$ of the time in a day.

Although the potential of developing wave power is promising [2,3], there is only a few full scale model remains at sea. The idea of commercializing wave farm is still in consideration due to the high operating cost compared to other RE sources. In addition, it is difficult to operate at the unpredictable

\footnotetext{
* Corresponding author.

E-mail address: kengwai.chan@usm.my (Keng Wai Chan)
}

https://doi.org/10.37934/cfdl.12.6.118133 
large force and oscillating motions of the wave which is used to drive the generator with a sufficient quality output for utilization [4]. In the long run, the performance of wave energy devices in saltwater will deteriorate and higher cost is needed for the maintenance [5].

Among the RE resources, wave energy has been overlooked to the extent of no commercial scale power plants. There are a few challenges that are needed to be considered before commercializing the wave energy harvesting to the global market. Effective wave energy collection device in a compact space is essential to maximize the output at any location at the sea. Efficiency of the devices can be improved if optimization had done according to location and wave condition [5-10].

The commercial utilization of wave energy requires the setup of huge amount of WEC in order to offer sufficient power to transform the variable input to electrical output [11-13]. In addition, deployment along with a remote converter with more than one body is also counted as a device array. These kinds of wave farms are alternative in bringing an enormous saving in terms of the cost of a mooring system, power transfer system, maintenance and other requirements. In contrast to the amount of numerical simulations of isolated WECs and numerous experimental works regarding WEC arrays, there is a small proportion of work in investigating numerical wave energy farms particularly in the time domain and 3D conditions [14-19].

The hydrodynamic interactions between the converter body of WEC and irregular waves which include waves that can be reflected or radiated from different converters are very complex. The hydrodynamic behavior among the devices may also have a positive and negative impact on the individual power output as compared with isolated installations and subsequently the overall power output from the array cannot easily be calculated by multiplying the number of devices by the power captured from the isolated device. Additionally, there are many elements which can impact the nature of this interaction, such as wave conditions, the shape of the device and power take-off system parameters.

As overall power output of the buoy system cannot easily be calculated and there are many parameters of the nature may impact the outcome. A computational fluid dynamic (CFD) simulation would be a reasonable and comparable solution compared to high cost experimental setup. In this paper, a wave structure interaction with application to wave energy device was studied numerically using Flow-3D. The CFD analysis based on the Reynolds Average Navier-Stokes (RANS) equations was used to investigate the interaction between wave and structure, and array effects among devices. Validation and predication of the performance of wave point absorber were done in this paper.

\section{Methodology}

Numerical models are built and simulations are run by using Flow-3D ( $v$ 11.2.4). Before the simulation of point absorber, validation on simulation of wave tank is done by comparing the simulation results with two external sources. After the validation is done, the simulation of point absorber in the waves is started by manipulating the characteristic dimension, radius, of different shapes of buoy. The characteristic dimension that yields the best result is used and set as the constant variable in the second part of simulation. In the second part, the manipulated variable is the mass of the buoy, where the performance of point absorber under constant characteristic dimension but different masses are studied. Finally, the shape of buoy that produce highest velocity and force are determined based on characteristic dimension equivalence and mass density analysis. 


\subsection{Based Modelling Validation}

A 3D simulation of wave tank with dimension $35.0 \mathrm{~m} \times 2.5 \mathrm{~m} \times 1.5 \mathrm{~m}$ is done by using Flow-3D. The results are validated by using two sources. The first validation source is the "Numerical Modelling of a Surging Point Absorber Wave Energy Converter" by Bhinder et al., [20]. Numerical Model with same parameters are constructed and imported into Flow 3-D. The second validation source is the "Numerical Simulation of Linear Water Waves and Wave-Structure Interactions" by Finnegan and Goggins [21]. The results obtained from the simulation are used to compare with the results from these sources.

\subsection{Design Modelling}

Five types of shape of buoy (shown in Figure 1) are considered in this study, which are the sphere, cylinder, cube, cone and pyramid. First, the mass of buoy is fixed at $0.138 \mathrm{~kg}$ and the buoys with characteristic dimension - radius, in this study, $0.10 \mathrm{~m}, 0.15 \mathrm{~m}$, and $0.20 \mathrm{~m}$ are simulated to determine the optimum value. Then the characteristic dimension is fixed at the optimum value and the buoy with mass of $0.138 \mathrm{~kg}, 1.38 \mathrm{~kg}$ and $13.8 \mathrm{~kg}$ are simulated. Volume of the buoy is determined through formula in Table 1.

(a)

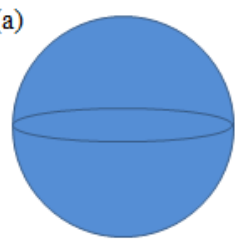

(b)

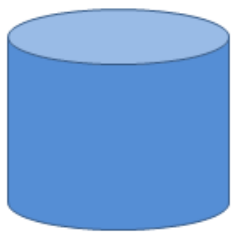

(c)

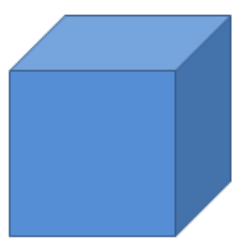

(d)

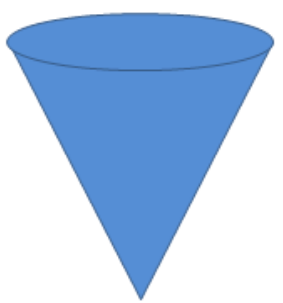

(e)

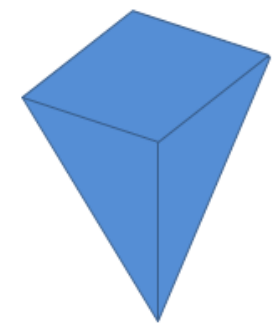

Fig. 1. Five different models used in this study (a) sphere (b) cylinder (c) cube (d) cone and (d) pyramid

Table 1

Volume of the shape

Buoy volume $\left(\mathrm{m}^{3}\right)$




\subsection{Numerical Modelling}

An implicit and the generalized minimum residual (GMRES) pressure solver option is chosen over explicit. The difference between an explicit and implicit solution is that an explicit is solved progressively at each computational cell by stepping through time, while the time step is restricted to meet stability criteria. An implicit solution, however is solved in each time step using information from another time step, something that requires more complex iterative or matrix solutions without imposing a time step restriction.

Split Lagrangian method is used in the volume of fluids (VOF) advection section of the Numerics tab as this method typically produces lower cumulative volume error than other methods available in Flow-3D.

All simulation is run while solving both momentum and continuity equations with second order monotonicity preserving selected based on information provided in the Flow-3D user's manual (2012). Renormalized group (RNG) model is used for all the simulations. The decision is based on the comments in Flow-3D user's manual 2012 that the RNG turbulence model is the most accurate and robust model available in the software (Flow Science, 2012) [22]. The summary of the simulation setup is shown in Table 2.

Table 2

Simulation properties details

\begin{tabular}{ll}
\hline Fluid Properties & \\
\hline Specific Heat (J/kg.K) & 4182 \\
Thermal Conductivity (W/m.K) & 0.597 \\
Viscosity $(\mathrm{kg} / \mathrm{m} . \mathrm{s})$ & 0.001 \\
Fluid Temperature $\left({ }^{\circ} \mathrm{C}\right)$ & 20 \\
Turbulence & Tabular \\
Density $\left(\mathrm{kg} / \mathrm{m}^{3}\right)$ & 1000 \\
\hline Simulation Properties & \\
\hline Wave height & $0.3 \mathrm{~m}$ \\
Water Depth & $1.5 \mathrm{~m}$ \\
Time Period & $4.2 \mathrm{~s}$ \\
Tank Dimensions & $35 \mathrm{~m} \times 1.5 \mathrm{~m} \times 2.5 \mathrm{~m}$ \\
Total Number of Cells & 793638 \\
Smallest Cell Size & 0.054773 \\
Pressure (Pa) & 101325 \\
Flow Model & Incompressible \\
Number of Fluid & One Fluid \\
Moving Object Model & Implicit \\
\hline Air Entrainment Model & Default \\
Gravitational acceleration (m/s ${ }^{2}$ ) & -9.81 in z-direction \\
Turbulent Model & Renormalized group (RNG) model \\
Pressure Solver & Implicit, GMRES \\
Volume of Fluid Advection & Split Lagrangian Method \\
Momentum Advection & Second order monotonically preserving \\
\hline
\end{tabular}

\subsubsection{General moving object (GMO)}

GMO model allows users to have multiple moving objects in one problem, and each moving object can have any independently defined type of motion. At each time step, the hydraulic force and torque due to pressure and shear stress are calculated. A body system $\left(x^{\prime}, y^{\prime}, z^{\prime}\right)$ is set up for each moving object with its coordinate axes parallel to those of the space system at time $t=0$. If an object 
motion has six DOF, the origin of the body system is set at the object mass center, $\mathrm{G}$. The body system is fixed on the moving object and experiences the same translation and rotation as the moving object. Equations of motion governing the two separate motions are

$F=m \frac{d V_{G}}{d t}$

$T_{G}=[J] \cdot \frac{d \omega}{d t}+\omega *([J] \cdot \omega)$

The total force and total torque are calculated as the sum of several components

$F=F_{g}+F_{h}+F_{c}$

$T_{G}=T_{g}+T_{h}+T_{c}$

where $F_{g}$ is the gravitational force, $F_{h}$ is the hydraulic force and $F_{c}$ is the net control force prescribed to control or restrict the rigid body motion. Similarly, $T_{G}, T_{g}, T_{h}$ and $T_{c}$ represent the total torque, gravitational torque, hydraulic torque and control torque about the mass center respectively.

\subsubsection{The renormalized group ( $R N G$ ) turbulence model}

The RNG turbulence model is derived from instantaneous Navier-Stokes equations. The analytical derivation result is a RNG model with constant different from those in the standard $k-\varepsilon$ model and additional terms and functions in the transport equations for $k$ and $\varepsilon$

$\frac{\partial}{\partial t}(\rho k)+\frac{\partial k}{\partial x_{i}}\left(\rho k u_{i}\right)=\frac{\partial}{\partial x_{j}}\left[\left(a_{k} u_{\varepsilon f f}\right) \frac{\partial k}{\partial x_{i}}\right]+G_{k}-\rho \varepsilon$

$\frac{\partial}{\partial t}(\rho \varepsilon)+\frac{\partial k}{\partial x_{i}}\left(\rho \varepsilon u_{i}\right)=\frac{\partial}{\partial x_{j}}\left[\left(a_{\varepsilon} u_{\varepsilon f f}\right) \frac{\partial \varepsilon}{\partial x_{i}}\right]+G_{l \varepsilon} \frac{\varepsilon}{k} G_{k}-G_{2 \varepsilon}^{*} \rho \frac{\varepsilon^{2}}{k}$

$G^{*}{ }_{2 \varepsilon}=G_{2 \varepsilon}+\frac{c_{\mu} \rho \eta^{3}\left(1-\frac{\eta}{\eta_{o}}\right)}{1+\beta \eta^{3}}$

$\eta=\frac{s k}{\varepsilon}$

\subsubsection{Meshing}

A nested mesh blocks was added surrounding the buoys as shown in Figure 2 to improve the resolution without excessively increasing the total number of cells. A nested mesh block is defined in Flow-3D as a mesh block that has smaller mesh size that lies within the boundaries of a surrounding mesh block. This technique enables the model to be more effectively capture the complete nested geometry and flow detail without increasing simulation times. 


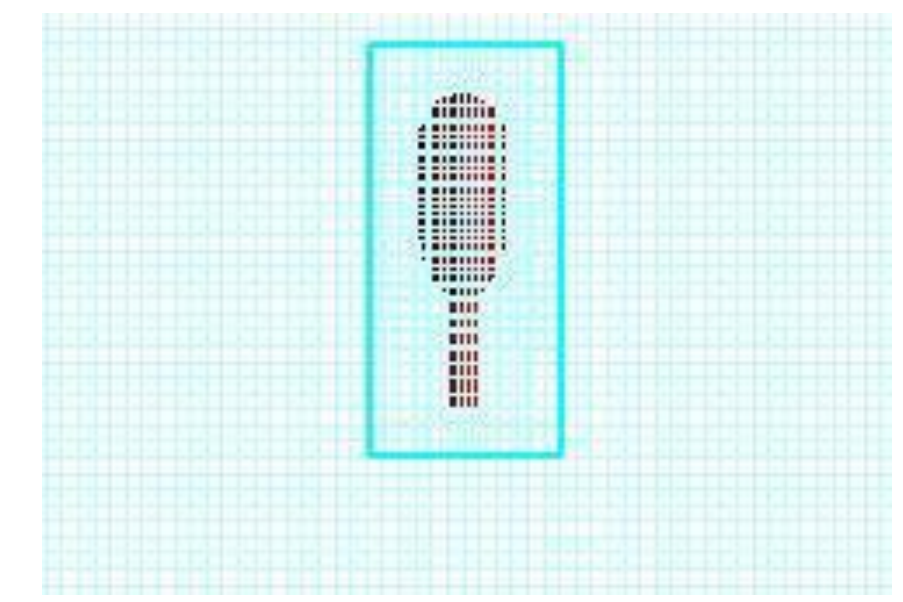

Fig. 2. Side view of the computational domain showing nested mesh blocks

Best mesh dimensions are determined before simulation by carrying out mesh independence tests. In these mesh independence tests, sizes of mesh tested in the simulation were 200000, 400000, 600000,800000 and 1000000 . The effect of each mesh on the velocity was shown in Figure 3 below.

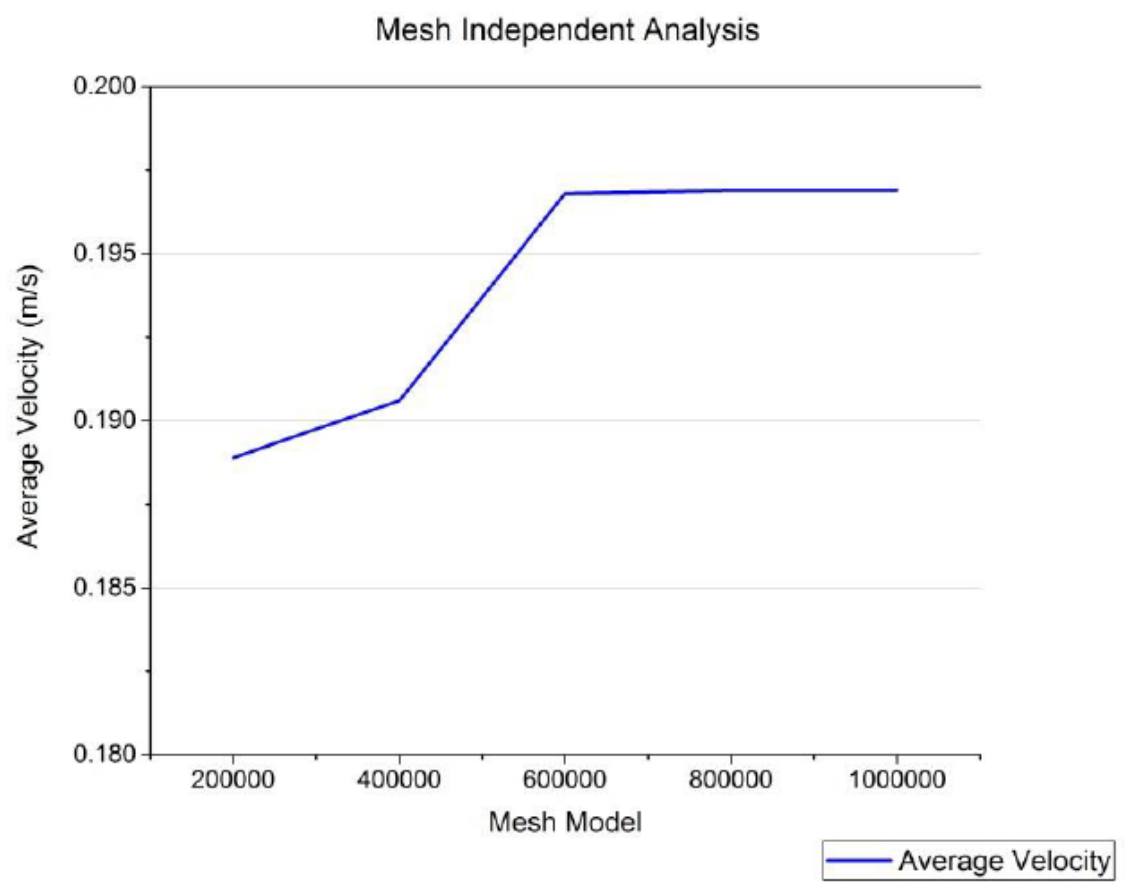

Fig. 3. Mesh independent analysis graph

From the graph, the most suitable mesh grid used in this simulation study is between 600000 to 800000 of total cells since it optimizes both the computation time and accuracy of the obtained solution. In the simulation, the number of mesh cells used is 793638 .

\subsubsection{Boundary and initial conditions}

The following boundary conditions (as in Figure 4 and Table 3) are based on "Numerical Modelling of a Surging Point Absorber Wave Energy Converter" by Bhinder et al., [20]. 
Table 3

The Boundary Conditions [20]

\begin{tabular}{lll}
\hline Face Number & Face of NWT & Boundary Condition \\
\hline 1 & Left (Upstream)- Min X & Wave Inlet \\
2 & Right (Downstream)- Max X & Outflow \\
3 & Front - Min Y & Symmetry \\
4 & Back - Max Y & Symmetry \\
5 & Bottom - Min Z & Wall \\
6 & Top - Max Z & Fixed Pressure \\
\hline
\end{tabular}

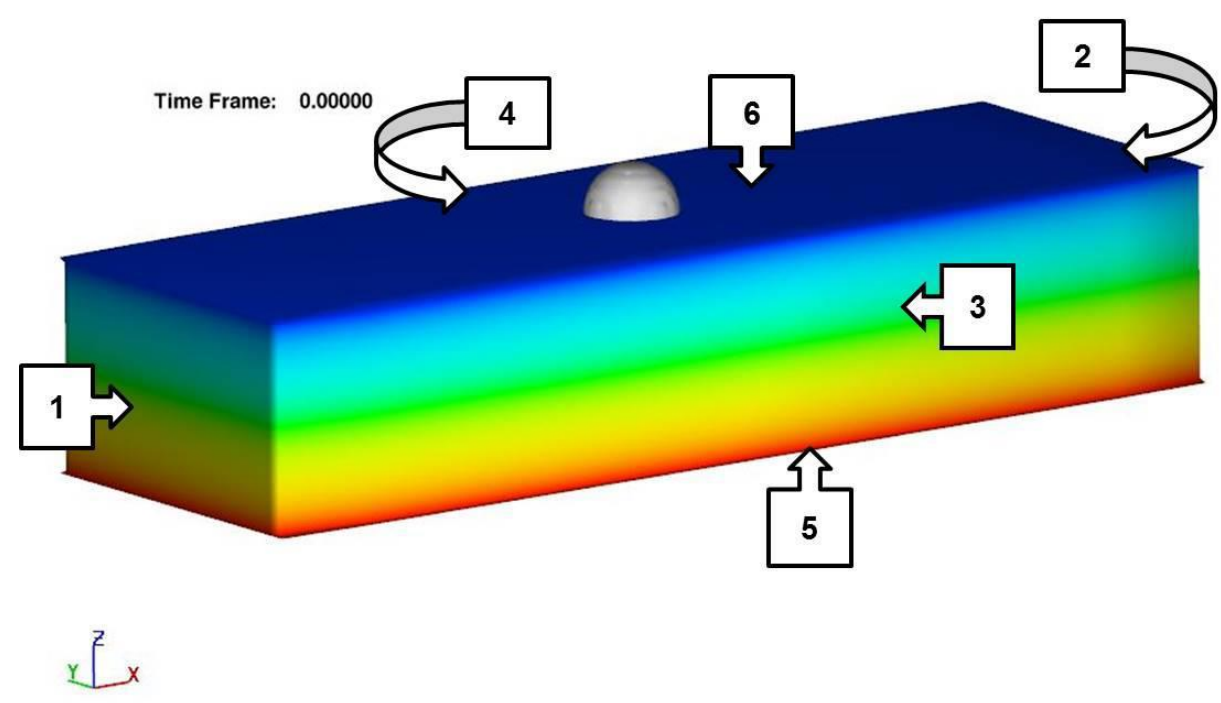

Fig. 4. The labels refer to the boundary conditions

\subsubsection{Fifth-Order Stokes Wave}

The fifth-order Stokes wave can be generated at the mesh boundary. The model is based on the fifth-order Stokes wave theory developed by Fenton [22]. As shown in Figure 5, a wave train is assumed to come from a flat bottom reservoir into the computational domain through the mesh boundary.

The wave is assumed to come from a flat bottom reservoir, which is outside the computational domain.

Wave propagation direction

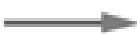

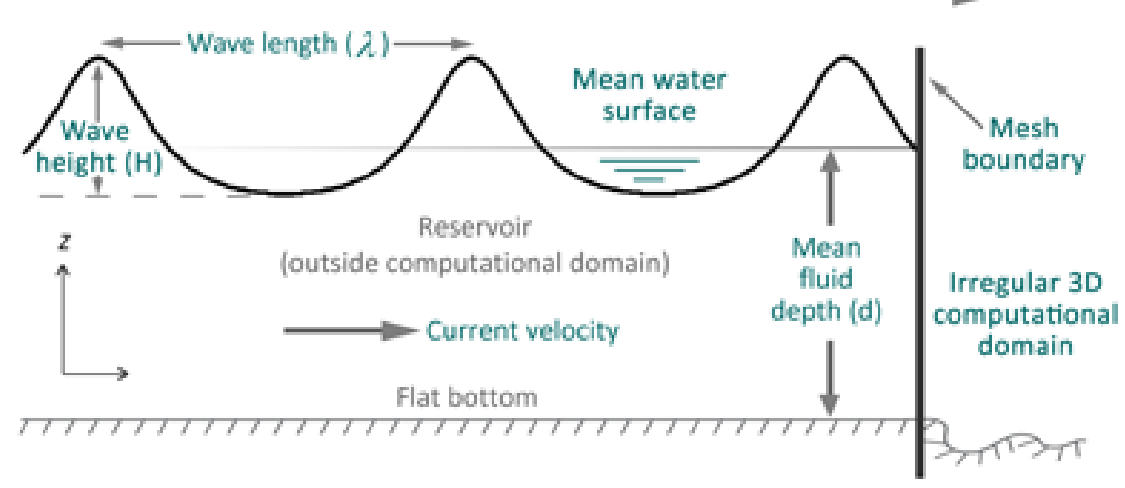

Fig. 5. Stokes wave definition [22] 
The reference system $(x, z)$ is established with its origin fixed at the bottom, $+x$ going in the wave propagation direction and $+z$ in the upward direction. The wave is characterized by the wave height $H$, wavelength, $\lambda$ and wave period $T$. The undisturbed water depth is $d=$ const. Water elevation, $\eta$ is time-dependent and measured along $+z$ from the bottom to the water surface. A current may exist and its $x$-component of undisturbed velocity is $U=$ const. The angular wave frequency, $\omega$ and the wave speed, $c$ are related to other parameters as

$\omega=\frac{2 \pi}{T}=c k \quad c=\frac{\lambda}{T}$

where the wave number, $k=\frac{2 \pi}{\lambda}$

The Stokes wave theory assumes potential flow, namely the fluid flow is incompressible and irrotational. The stream function, $\psi$ thus exists and satisfies the Laplace equation

$\nabla^{2} \varphi=0$

Fluid velocity components in $x$ and $\underline{z}$ are given by

$u=\frac{\partial \varphi}{\partial x}$ and $v=\frac{\partial \varphi}{\partial z}$

With the further assumption that a wave crest exists at $x=0$ at $t=0$, the Laplace equation for $\varphi$, along with its boundary conditions at the free surface and the bottom, are solved using a perturbation method. The perturbation parameter is the dimensionless wave amplitude, $\varepsilon=k H / 2$, also known as the wave steepness. The solution for the water elevation and velocity with fifth-order accuracy with respect to $\boldsymbol{\varepsilon}$ is

$\eta(x, t)=d+\frac{\varepsilon}{k} \cos k X+\frac{\varepsilon^{2}}{k} B_{22} \cos 2 k X+\frac{\varepsilon^{3}}{k} B_{31}(\cos k X-\cos 3 k X)+\frac{\varepsilon^{4}}{k}\left(B_{42} \cos 2 k X-\right.$

$\left.B_{44} \cos 4 k X\right)+\frac{\varepsilon^{5}}{k}\left[-\left(B_{53}+B_{55}\right) \cos k X+B_{53} \cos 3 k X+B_{53} \cos 5 k X\right]$

$u=U+C_{o}\left(\frac{g}{k^{3}}\right)^{\frac{1}{2}} \sum_{i=1}^{5} \varepsilon^{i} \sum_{j=1}^{i} A_{i j} j k \cosh j k z \cos j k X$

$w(x, z, t)=C_{o}\left(\frac{g}{k^{3}}\right)^{\frac{1}{2}} \sum_{i=1}^{5} \varepsilon^{i} \sum_{j=1}^{i} A_{i j} j k \sinh j k z \sin j k X$

where $X=x-c t, k X=k x-\omega t, A_{i j}, B_{i j}$ and $C_{o}$ are non linear function of $k d$. It can be seen that the linear wave theory is the first-order approximation of the Stokes theory. In other words, the fifthorder Stokes theory is four-orders of $\varepsilon$ higher in accuracy than the linear wave theory. The wave number and wave frequency are non-independent parameters but satisfy the nonlinear equation.

$\left(\frac{k}{g}\right)^{1 / 2} U-\frac{\omega}{(g k)^{\frac{1}{2}}}+C_{0}+\left(\frac{k H}{2}\right)^{2} C_{2}+\left(\frac{k H}{2}\right)^{4} C^{4}=0$

where $C_{0}, C_{2}$ and $C_{4}$ are nonlinear functions of $k d$. It is found that the dispersion equation for linear wave is the first-order approximation of this relation without considering the last two terms at the left-hand side. As a result of this relation, when generating wave at a mesh boundary, the users 
cannot provide both wave length and wave period as input parameters at the same time. If a wave length is given, then the wave period is obtained easily from this equation. If wave period is known, instead, the wave length is calculated from the above equation iteratively. The first approximation of $k$ used in the iterations is

$k=\frac{\alpha+\beta^{2} \operatorname{sech}^{2} \beta}{\left(\tan \beta+\beta \operatorname{sech}^{2} \beta\right) d}$

where $\alpha=\frac{\omega^{2} d}{g}$ and $\beta=\alpha \sqrt{\operatorname{coth} \alpha}$. This theory is applicable to waves in both deep and shallow water, but restrictions exist. The parameter $\varepsilon$ must be a small number, namely the wave's steepness must be small. Furthermore, the theory cannot work for waves that are long compared to the water depth.

\section{Results}

\subsection{Validation Results of Baseline Modelling}

For the validation of the modelling, two validation sources have been used. The first source is the study of Bhinder [20], and the second source is the study of Finnegan and Goggins [21]. These two sources were used because they used the same simulation programme for their buoy study.

\subsubsection{Validation source 1: Bhinder et al., [20]}

By using the same parameters with Bhinder's research, a linear wave was modelled to obtain the graph of free surface elevation against the finish time in seconds. Three highest peak values for each case are used for comparison, the difference is found to be less than $10 \%$ (as shown in Table 4). This slight difference is due to other setting which is not specified in the research and the geometry details are not clear.

Table 4

Percentage difference of the free surface elevation at $x=2.1 \mathrm{~m}$ and $\mathrm{x}=6.5 \mathrm{~m}$

\begin{tabular}{lllll}
\hline$X(m)$ & Highest Peak & \multicolumn{2}{l}{ Free Surface Evaluation $(m)$} & Percentage Difference (\%) \\
\cline { 3 - 4 } & & Baseline Model & Simulation Results & \\
\hline 2.1 & Peak 1 & 0.18 & 0.17 & 5.71 \\
& Peak 2 & 0.19 & 0.18 & 5.41 \\
& Peak 3 & 0.185 & 0.175 & 5.55 \\
6.5 & Peak 1 & 0.155 & 0.155 & 0 \\
& Peak 2 & 0.155 & 0.145 & 6.67 \\
& Peak 3 & 0.145 & 0.14 & 3.51 \\
\hline
\end{tabular}

Figure 6 illustrates that the comparison of simulation results based on plotted graph. 


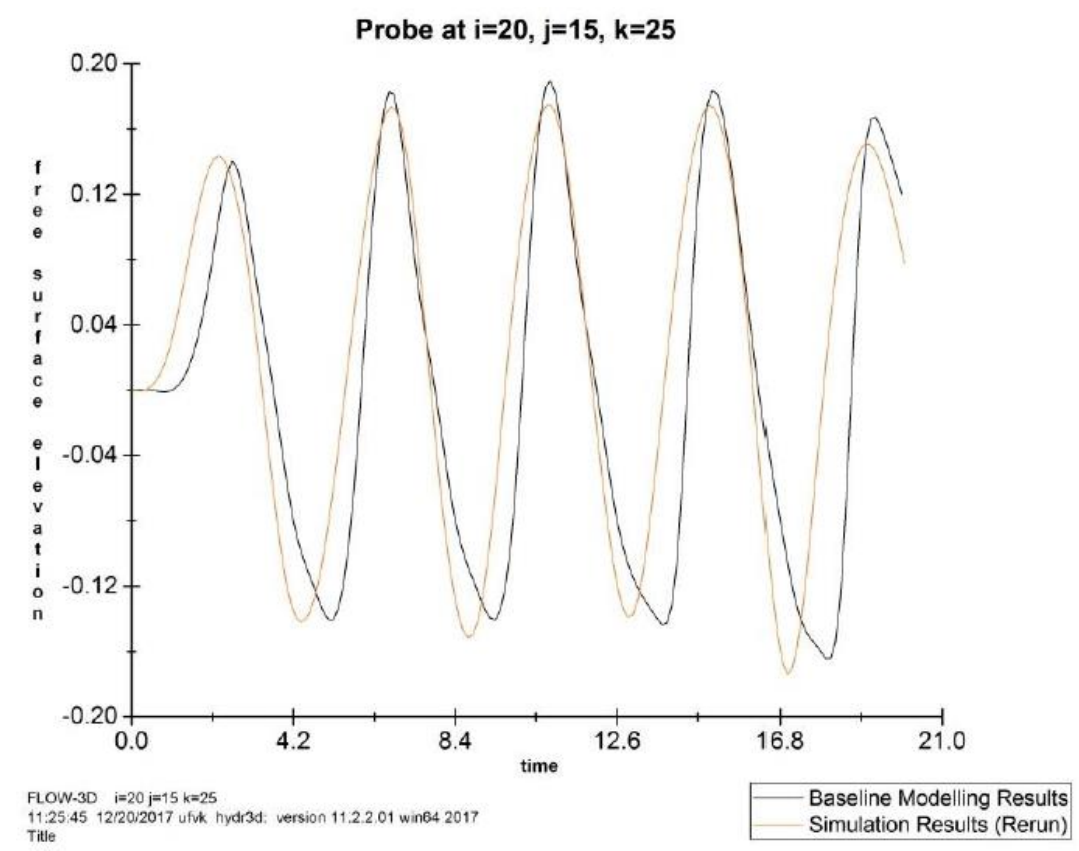

Fig. 6. Free surface elevation of at $x=2.1 \mathrm{~m}$

\subsubsection{Validation source 2: Finnegan and Goggins [21]}

CFD analysis is done to obtain the graph of wave displacement against time. The graph is validated by comparing the results with the model described in the study of Finnegan \& Goggins. By comparing Figure 7 and Figure 8 , it can be seen that both graphs have close pattern. The difference can be explained by different size of the buoy used in both the research.

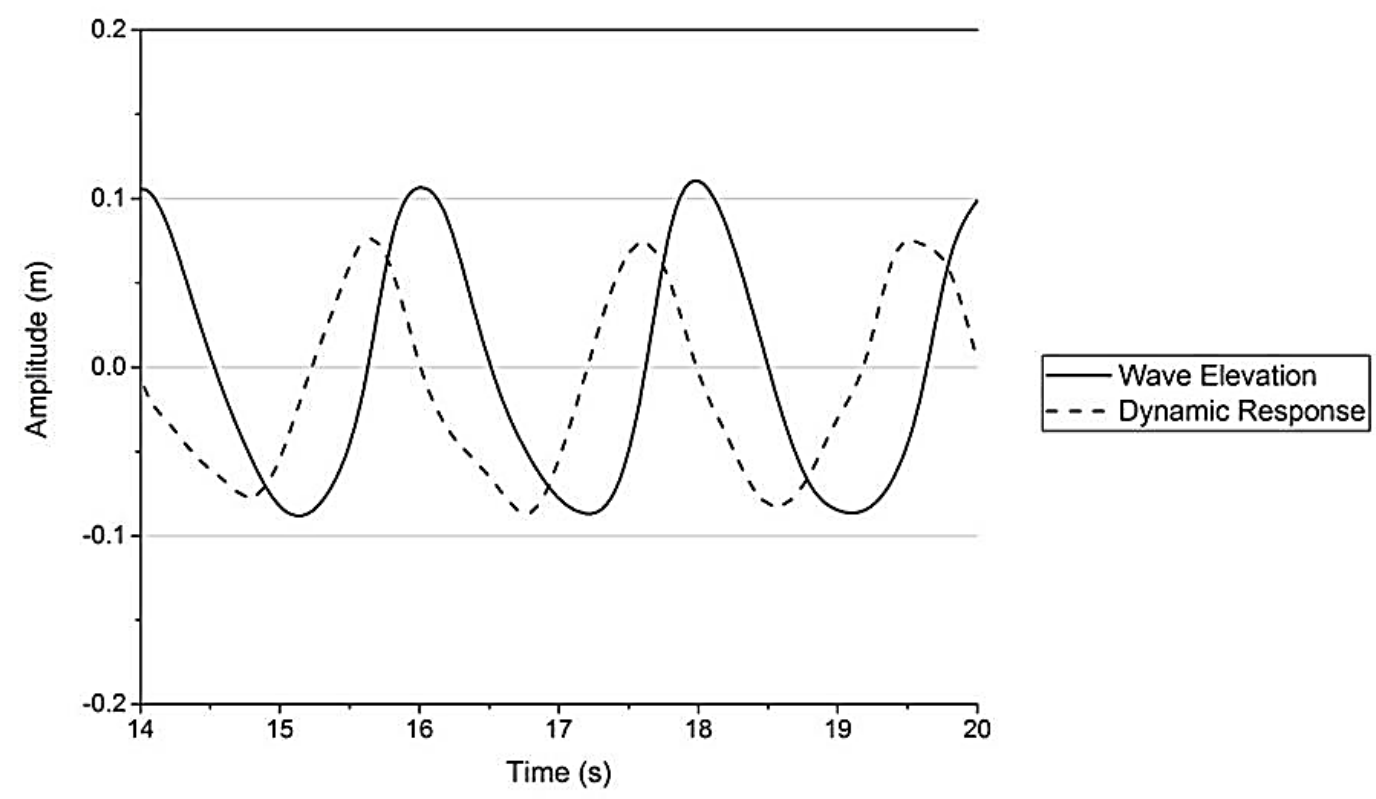

Fig. 7. Wave elevation and the dynamic response of the floating buoy in heave motion 


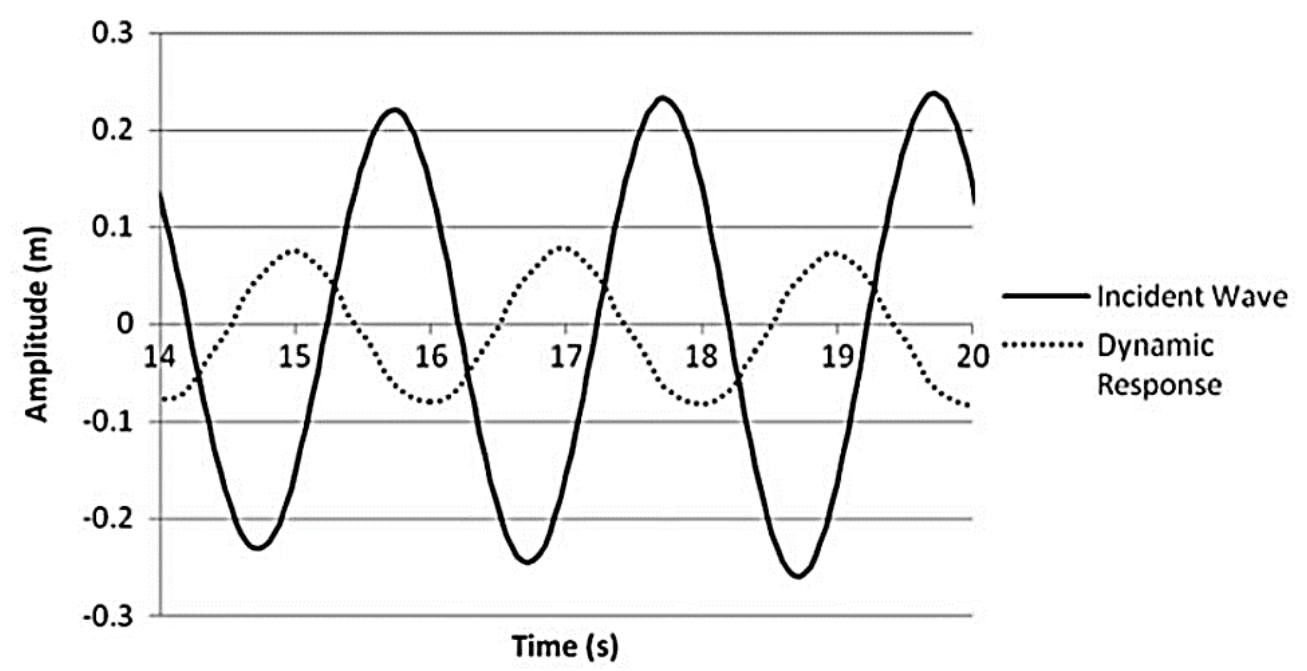

Fig. 8. Wave elevation and the dynamic response of the floating cylibe buoy in heave motion (Finnegan 7 Googins, 2012)

Three highest peaks are chosen for percentage difference calculation. From Table 5 it can be seen that the percentage difference for both the results is less than $10 \%$. Therefore, numerical simulation using Flow-3D for this research is valid.

Table 5

Percentage difference of dynamic response amplitude

\begin{tabular}{llll}
\hline Highest Peak & \multicolumn{2}{l}{ Dynamic Response Amplitude $(\mathrm{m})$} & Percentage Difference (\%) \\
\cline { 2 - 3 } & Baseline Model & Simulation Results & \\
\hline Peak 1 & 0.08 & 0.085 & 6.06 \\
Peak 2 & 0.08 & 0.085 & 6.06 \\
Peak 3 & 0.08 & 0.08 & 0 \\
\hline
\end{tabular}

\subsection{Effect of Buoy Characteristic Radius}

By fixing the mass of buoy at $0.138 \mathrm{~kg}$, the effect of buoy characteristic dimension on its average velocity is studied for different shapes of buoy as shown in Table 6 .

Table 6

Average velocity for $0.138 \mathrm{~kg}$ buoy of different characteristic radius

\begin{tabular}{lll}
\hline Shape & Radius $(\mathrm{m})$ & Average Velocity $(\mathrm{m} / \mathrm{s})$ \\
\hline Sphere & 0.10 & 0.1967 \\
& 0.15 & 0.1968 \\
Cube & 0.20 & 0.2077 \\
& 0.10 & 0.2306 \\
Cylinder & 0.15 & 0.2310 \\
& 0.20 & 0.2355 \\
Cone & 0.10 & 0.2327 \\
& 0.15 & 0.2330 \\
& 0.20 & 0.2430 \\
Pyramid & 0.10 & 0.2275 \\
& 0.15 & 0.2279 \\
& 0.20 & 0.2293 \\
& 0.10 & 0.2018 \\
& 0.15 & 0.2063 \\
\end{tabular}


From the table, it can be said that the buoy with a larger dimension yields a higher velocity for all the shapes. The average velocity of buoy is highest when the radius of buoy is $0.2 \mathrm{~m}$. Among the shapes of buoy with radius $0.2 \mathrm{~m}$, cylinder buoy has highest velocity of $0.2430 \mathrm{~m} / \mathrm{s}$ sphere has lowest velocity of $0.2077 \mathrm{~m} / \mathrm{s}$. High velocity indicates a larger kinetic energy from wave motion is transferred to the buoy and then to be converted into electrical energy through PTO system. Figure 9 illustrates the graph of velocity of cylindrical buoy with different radius against time.

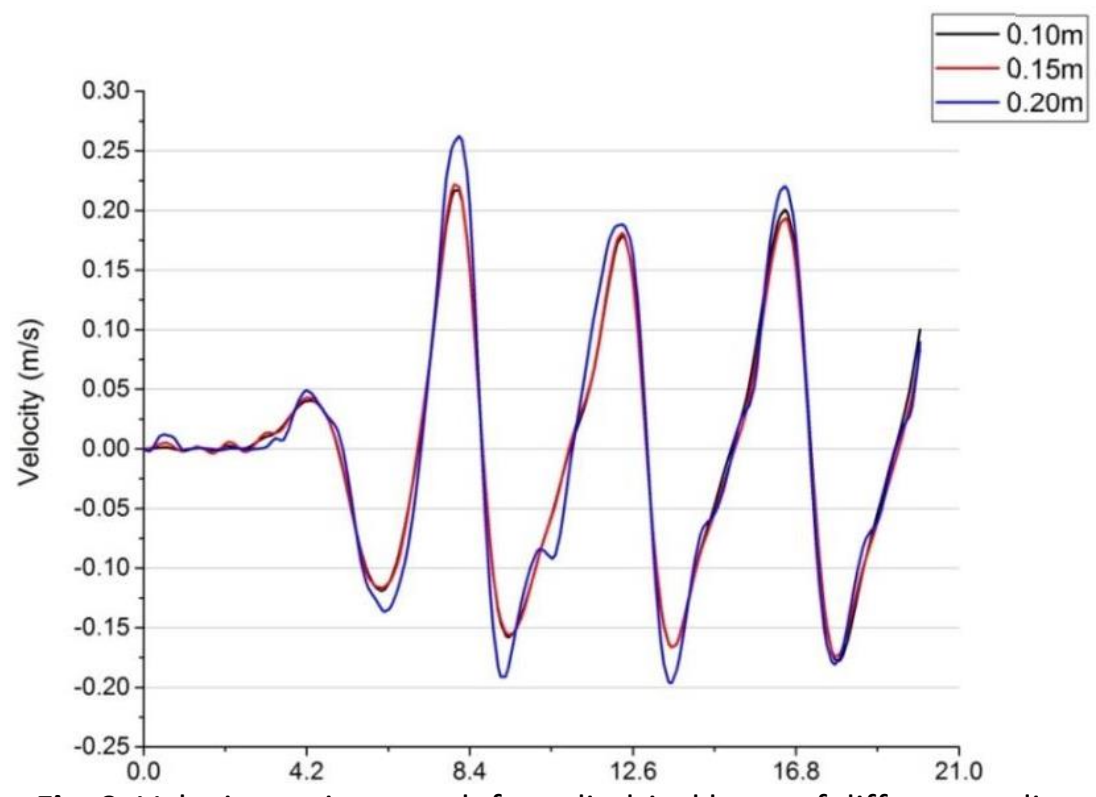

Fig. 9. Velocity vs time graph for cylindrical buoy of different radius

\subsection{Effect of Buoy Mass}

By fixing the radius of buoy at $0.2 \mathrm{~m}$, the average velocity and the force produced by the buoy are studied by manipulating the mass of buoy. The mass of buoy being used in the simulation are $0.138 \mathrm{~kg}$, $1.38 \mathrm{~kg}$ and $13.8 \mathrm{~kg}$. The results of average velocity of buoy with different shape and mass are tabulated in Table 7.

Table 7

Average velocity for characteristic radius $0.2 \mathrm{~m}$ buoy of different mass

\begin{tabular}{lll}
\hline Shape & Mass $(\mathrm{kg})$ & Average Velocity $(\mathrm{m} / \mathrm{s})$ \\
\hline Sphere & 0.138 & 0.2077 \\
& 1.38 & 0.2494 \\
Cylinder & 13.8 & 0.2517 \\
& 0.138 & 0.2430 \\
Cube & 1.38 & 0.2506 \\
& 13.8 & 0.3395 \\
& 0.138 & 0.2355 \\
Cone & 1.38 & 0.2487 \\
& 13.8 & - (the buoy sinks) \\
& 0.138 & 0.2293 \\
Pyramid & 1.38 & 0.2502 \\
& 13.8 & - (the buoy sinks) \\
& 0.138 & 0.2145 \\
& 1.38 & 0.2408 \\
& 13.8 & - (the buoy sinks)
\end{tabular}


The average velocity of buoy increases when the mass of buoy increase. However, for the cases of cube, cone and pyramid, the buoy sinks at $13.8 \mathrm{~kg}$ because its density exceed $1000 \mathrm{~kg} / \mathrm{m}^{3}$ which is the density of water. The mass density for cube, cone, and pyramid at $13.8 \mathrm{~kg}$ with characteristic radius $0.2 \mathrm{~m}$ is $1725 \mathrm{~kg} / \mathrm{m}^{3}, 1647.25 \mathrm{~kg} / \mathrm{m}^{3}, 5175 \mathrm{~kg} / \mathrm{m}^{3}$ respectively. Among all the shape, cylinder perform the best with highest mass, which it yields average velocity of $0.3395 \mathrm{~m} / \mathrm{s}$ at mass $13.8 \mathrm{~kg}$. Figure 10 shows the graph of velocity against time by cylindrical buoy at different mass.

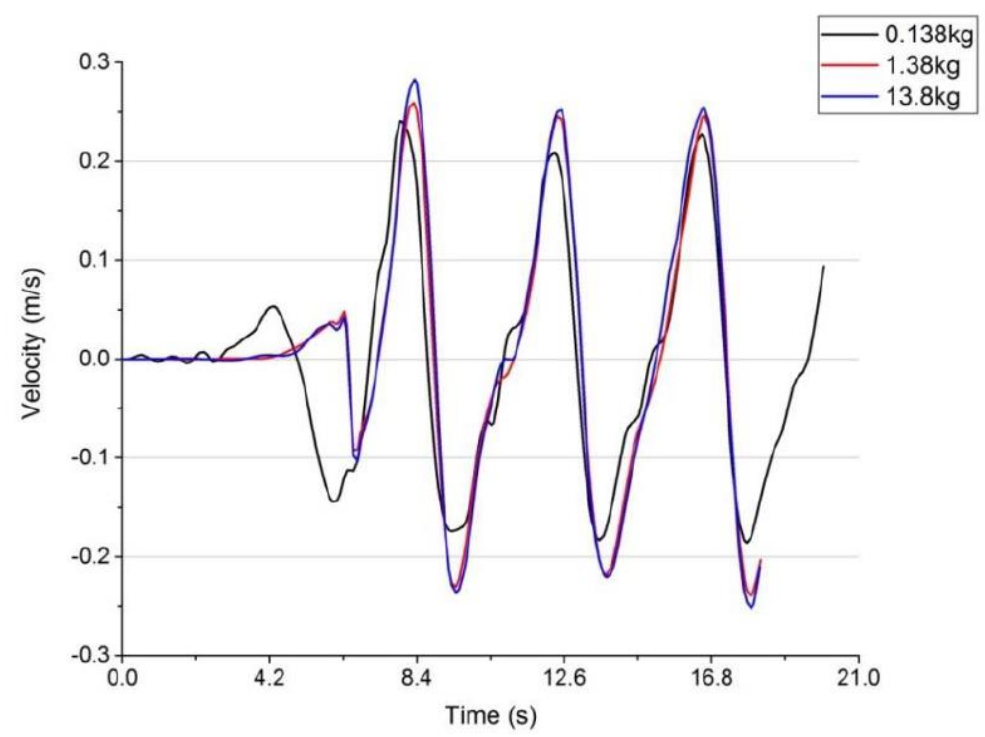

Fig. 10. Velocity vs time graph for cylindrical buoy of different mass

In terms of force production, the cylindrical buoy also has the best performance, as illustrated in Figure 11. It captures about $8.8 \%$ more force than the sphere shape buoy model of largest mass, but the difference at the smaller mass is too small to be noticed. The maximum force for cube, pyramid and cone is ignored due to the force for these three shapes cannot be taken into consideration because the buoy sinks to the bottom of the sink and the force is used to overcome the pressure of the water as the buoy sinks to the bottom of the tank.

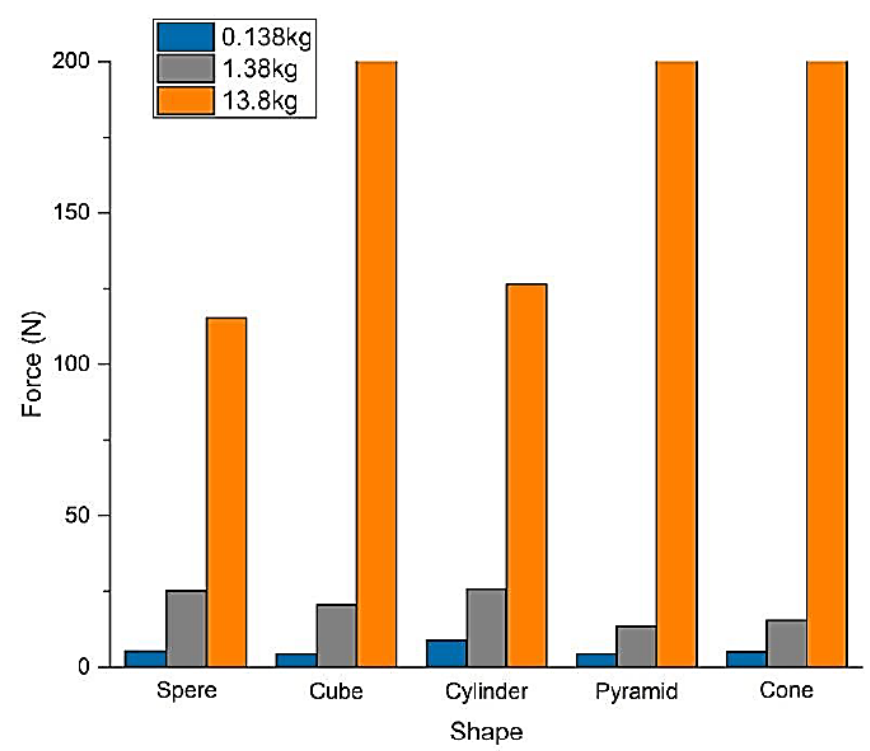

Fig. 11. Maximum force for all the shapes 


\subsection{Velocity Profile for Different Mass Density}

Since the simulation results have shown that the cylindrical buoy has the best performance among all shapes, its velocity profile is studied. Figure 12 below shows the velocity versus mass density graph for cylinder

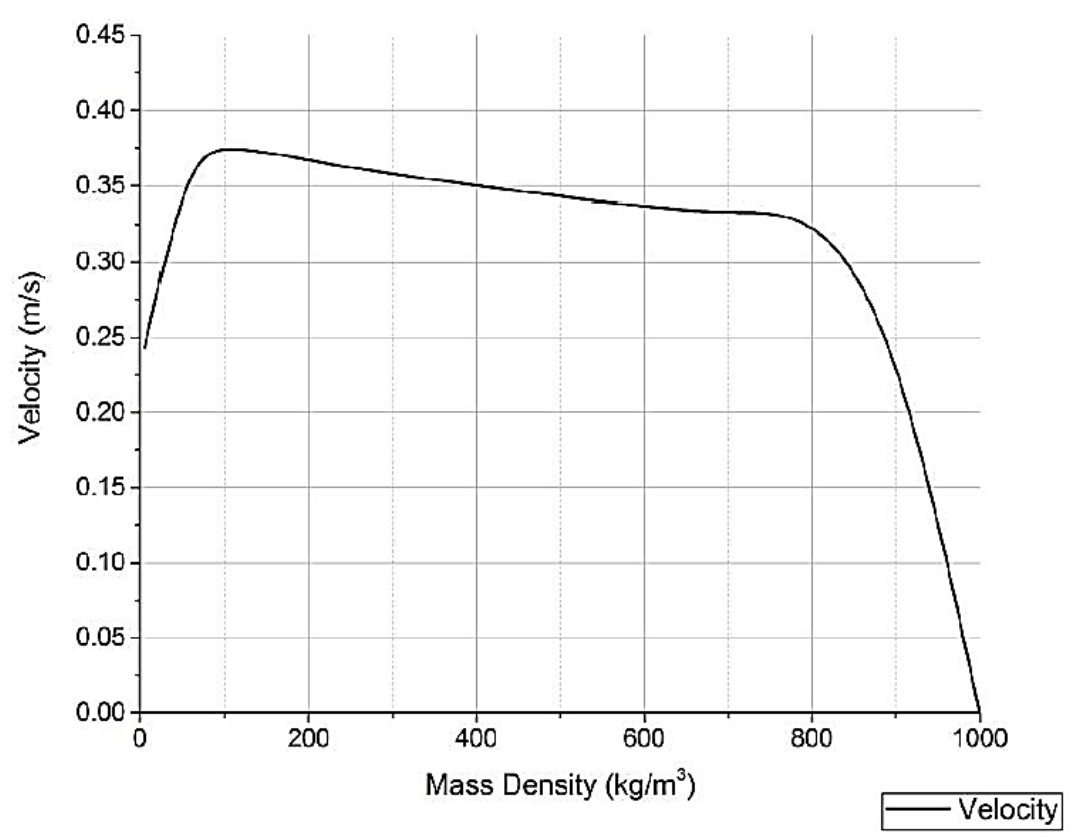

Fig. 12. Velocity vs mass density graph for cylinder

The peak of the graph is observed when cylindrical buoy has density of $100 \mathrm{~kg} / \mathrm{m}^{3}$, which is equivalent to $20 \mathrm{~kg}$ buoy with a radius of 0.20 .

\section{Conclusions}

In conclusion, the simulation results obtained by using Flow-3D are successfully validated by comparing the results with study of Bhinder et al., and Finnegan and Goggins. In both validations, the differences of results between simulation and validation sources are found to fall in the percentage of less than $10 \%$.

When the mass of buoy is fixed constant at $0.138 \mathrm{~kg}$, it is found that the average velocity of buoy increases as the radius of buoy increases. For all shapes, the buoy has highest velocity when the characteristic radius is $0.2 \mathrm{~m}$. Compared to other shapes of buoy, cylindrical buoy has optimum performance, with average velocity of $0.2430 \mathrm{~m} / \mathrm{s}$ at $0.2 \mathrm{~m}$ radius.

After the optimum value of radius is obtained, the simulation is continued by manipulating the mass of buoy. It is found that the average velocity of buoy increases as the mass of buoy increases, given the condition where the density of buoy is less than the density of water. Compared to other buoys, the cylindrical buoy gives the best performance, with average velocity of $0.3395 \mathrm{~m} / \mathrm{s}$ when the mass is $13.8 \mathrm{~kg}$. It has the best performance in force production.

By analysing the velocity profile of cylindrical buoy based on its mass density, it is found that it has optimum performance when the density is $100 \mathrm{~kg} / \mathrm{m}^{3}$, which is equivalent to $20 \mathrm{~kg}$ buoy with a radius of 0.20 . 


\section{Acknowledgement}

This research was supported by a grant from Ministry of Higher Education of Malaysia (Grant No. 311.PREDAC.4403901).

\section{References}

[1] Galarraga, Ibon, Mikel González-Eguino, and Anil Markandya, eds. Handbook of sustainable energy. Edward Elgar Publishing, 2011.

https://doi.org/10.4337/9780857936387

[2] Arent, Douglas J., Alison Wise, and Rachel Gelman. "The status and prospects of renewable energy for combating global warming." Energy Economics 33, no. 4 (2011): 584-593.

https://doi.org/10.1016/i.eneco.2010.11.003

[3] Oh, Tick Hui, Shen Yee Pang, and Shing Chyi Chua. "Energy policy and alternative energy in Malaysia: issues and challenges for sustainable growth." Renewable and Sustainable Energy Reviews 14, no. 4 (2010): 1241-1252.

https://doi.org/10.1016/i.rser.2009.12.003

[4] Drew, Benjamin, Andrew R. Plummer, and M. Necip Sahinkaya. "A review of wave energy converter technology." (2009): 887-902.

https://doi.org/10.1243/09576509JPE782

[5] Czech, Balazs, and Pavol Bauer. "Wave energy converter concepts: Design challenges and classification." IEEE Industrial Electronics Magazine 6, no. 2 (2012): 4-16. https://doi.org/10.1109/MIE.2012.2193290

[6] Cai, Ling. "Performance evaluation and parametric optimum design of an updated ocean thermal energy conversion system." Ocean Engineering 117 (2016): 254-258.

https://doi.org/10.1016/j.oceaneng.2016.03.026

[7] Chong, Heap-Yih, and Wei-Haur Lam. "Ocean renewable energy in Malaysia: The potential of the Straits of Malacca." Renewable and Sustainable Energy Reviews 23 (2013): 169-178.

https://doi.org/10.1016/j.rser.2013.02.021

[8] Nithesh, K. G., and Dhiman Chatterjee. "Numerical prediction of the performance of radial inflow turbine designed for ocean thermal energy conversion system." Applied Energy 167 (2016): 1-16.

https://doi.org/10.1016/i.apenergy.2016.01.033

[9] Hooi-Siang, Kang, Mohd Qisti Hakim Shaharuddin, Lee Kee-Quen, Adelina Steven, Ummul Ghafir Md Arif, Nurhaslinda Khaliddin, and Siow Chee-Loon. "Numerical Analysis of Point Absorber for Wave Energy Conversion in Malaysian Seas." Progress in Energy and Environment 1, no. 1 (2017): 25-39.

[10] Aiman, Muhamad Jalani, Nur Izzati Ismail, Mohd Rashdan Saad, Yasutaka Imai, Shuichi Nagata, Mohd Kamarul Huda Samion, Ernie Abd Manan, and Mohd Rosdzimin Abdul Rahman. "Study on Shape Geometry of Floating Oscillating Water Column Wave Energy Converter for Low Heave Wave Condition." Journal of Advanced Research in Fluid Mechanics and Thermal Sciences 70, no. 2 (2020): 124-134.

https://doi.org/10.37934/arfmts.70.2.124134

[11] Clément, Alain, Pat McCullen, António Falcão, Antonio Fiorentino, Fred Gardner, Karin Hammarlund, George Lemonis et al. "Wave energy in Europe: current status and perspectives." Renewable and sustainable energy reviews 6 , no. 5 (2002): 405-431.

https://doi.org/10.1016/S1364-0321(02)00009-6

[12] Retzler, Chris. "Measurements of the slow drift dynamics of a model Pelamis wave energy converter." Renewable Energy 31, no. 2 (2006): 257-269.

https://doi.org/10.1016/i.renene.2005.08.025

[13] António, F. de O. "Modelling and control of oscillating-body wave energy converters with hydraulic power take-off and gas accumulator." Ocean engineering 34, no. 14-15 (2007): 2021-2032. https://doi.org/10.1016/i.oceaneng.2007.02.006

[14] Bhinder, Majid A., Clive G. Mingham, Derek M. Causon, Mohammad T. Rahmati, George A. Aggidis, and Robert V. Chaplin. "A joint numerical and experimental study of a surging point absorbing wave energy converter (WRASPA)." In ASME 2009 28th International Conference on Ocean, Offshore and Arctic Engineering, pp. 869-875. American Society of Mechanical Engineers Digital Collection, 2009.

https://doi.org/10.1115/OMAE2009-79392

[15] Westphalen, J., D. M. Greaves, Alison Hunt-Raby, Christopher JK Williams, Paul H. Taylor, Z. Z. Hu, D. M. Causon et al. "Numerical simulation of wave energy converters using Eulerian and Lagrangian CFD methods." In The Twentieth International Offshore and Polar Engineering Conference. International Society of Offshore and Polar Engineers, 2010. 
[16] Didier, E., JM Paixão Conde, and P. R. F. Teixeira. "Numerical simulation of an oscillating water column wave energy convertor with and without damping." In Proc., International Conference on Computational Methods in Marine Engineering, pp. 206-217. 2011.

[17] Schmitt, P., K. Doherty, D. Clabby, and T. Whittaker. "The opportunities and limitations of using CFD in the development of wave energy converters." Marine \& Offshore Renewable Energy (2012): 89-97.

[18] Rafiee, Ashkan, Bjoern Elsaesser, and Frederic Dias. "Numerical simulation of wave interaction with an oscillating wave surge converter." In ASME 2013 32nd International Conference on Ocean, Offshore and Arctic Engineering. American Society of Mechanical Engineers Digital Collection, 2013. https://doi.org/10.1115/OMAE2013-10195.

[19] Li, L., M. Tan, and J. I. R. Blake. "Numerical simulation of multi-body wave point-absorber." CRC Press, (2015): $723-$ 730. https://doi.org/10.1201/b18179-94

[20] Bhinder, M. A., C. G. Mingham, D. M. Causon, M. T. Rahmati, G. A. Aggidis, and R. V. Chaplin. "Numerical modelling of a surging point absorber wave energy converter." In Proc. of 8th European Wave and Tidal Energy Conference EWTEC. 2009.

[21] Finnegan, William, and Jamie Goggins. "Numerical simulation of linear water waves and wave-structure interaction." Ocean Engineering 43 (2012): 23-31. https://doi.org/10.1016/j.oceaneng.2012.01.002

[22] FlowScience. "Flow-3D Documentation: Release 10.1." (2012): 813. 